\title{
GCU
}

Glasgow Caledonian

University

University for the Common Good

\section{Design of improved IR protocol for LED indoor positioning system}

\author{
Popoola, Olaoluwa R.; Popoola, Wasiu O.; Ramirez-Iniguez, Roberto; Sinanovic, Sinan
}

Published in:

2017 13th International Wireless Communications and Mobile Computing Conference, IWCMC 2017

DOI:

10.1109/IWCMC.2017.7986402

Publication date:

2017

Document Version

Author accepted manuscript

Link to publication in ResearchOnline

Citation for published version (Harvard):

Popoola, OR, Popoola, WO, Ramirez-Iniguez, R \& Sinanovic, S 2017, Design of improved IR protocol for LED indoor positioning system. in 2017 13th International Wireless Communications and Mobile Computing Conference, IWCMC 2017. IEEE, pp. 882-887, 13th International Wireless Communications and Mobile Computing Conference (IWCMC), Valencia, Spain, 26/06/17. https://doi.org/10.1109/IWCMC.2017.7986402

\section{General rights}

Copyright and moral rights for the publications made accessible in the public portal are retained by the authors and/or other copyright owners and it is a condition of accessing publications that users recognise and abide by the legal requirements associated with these rights.

Take down policy

If you believe that this document breaches copyright please view our takedown policy at https://edshare.gcu.ac.uk/id/eprint/5179 for details of how to contact us. 


\title{
Design of improved IR protocol for LED indoor positioning system
}

\author{
Olaoluwa R. Popoola*, Wasiu O. Popoola ${ }^{\dagger}$, Roberto Ramirez-Iniguez* and Sinan Sinanović* \\ ${ }^{*}$ School of Engineering and Built Environment, Glasgow Caledonian University, Glasgow, G4 0BA, UK \\ Email: \{olaoluwa.popoola, roberto.ramireziniguez, sinan.sinanovic\}@gcu.ac.uk ${ }^{\dagger}$ Institute for Digital \\ Communications, University of Edinburgh, Edinburgh, EH9 3JL, UK \\ Email:W.Popoola@ed.ac.uk
}

\begin{abstract}
In this work, we design an infrared protocol (IRP) for light emitting diode (LED) based indoor positioning. The designed IRP compensates for the shortcomings of other existing protocols when applied to the multiple LED estimation indoor positioning model (MLEM). MLEM uses overlap of LED beams to increase accuracy of positioning. The overlap sets up a multipoint-to-point optical communication channel. The existing protocols which are designed for point-to-point links, when modified to suit the MLEM overlapping region, show a high positioning time between $3 \mathrm{~s}$ and $4.5 \mathrm{~s}$. These values are not desirable for real time tracking. A new protocol is therefore designed to reduce the positioning time. The protocol is implemented in an experimental MLEM design using ATmega 328 microcontroller hardware. The experimental results show the new protocol reduces the positioning time to $0.5 \mathrm{~s}$.
\end{abstract}

Keywords- Indoor localization; optical wireless communications; multiple LEDs; microcontroller-based positioning; infrared protocols; overlap

\section{INTRODUCTION}

The use of light emitting diodes (LEDs) as both lighting and data transmitting devices has made LEDs a viable technology for indoor positioning systems (IPS). A number of research investigations into the use of LEDs in indoor positioning have been carried out [1]. Popular methods used in LED-based IPS include positioning based on received signal strength (RSS) [2], positioning by the use of fingerprint index or patterns [3], proximity-based positioning [4], positioning by image sensors [5], or a hybrid of any of these. Although earliest LEDbased IPS are designed by the proximity-based positioning method, the accuracy of systems based on this method is limited by the size of an enclosure or the footprint of the LED [4]. Due to this reduced accuracy of the proximity-based positioning methods, investigations of LED-based positioning by RSS, image positioning and fingerprinting have become more popular [6].

RSS based indoor positioning replicates the trilateration algorithm that has been successfully implemented in the popular global positioning system (GPS), with a view to obtaining similar accuracy figures indoors as GPS provides outdoors. However the peculiar characteristics of light beam such as the directional radiation pattern produced by LEDs and the additive property of optical signals result in an appreciable amount of hardware sophistication required for its implementation [7], [8]. A similar scenario applies to fingerprinting and image sensor based positioning systems [9]. In the latter, images of different light sources are collected on a photographic film or an image sensor. The sensor decodes the information sent from the LED so as to predict the position of a receiver. Some image based localization systems require the use of expensive cameras that inflate the cost of installing and maintaining the system in addition to causing issues with privacy.

Although RSS, fingerprinting and image based LED positioning systems have been shown to improve the positioning accuracy, the high economic and computational cost of implementing these systems hinders their real life applicability. In light of this, the drawback in proximity-based IPS is addressed in [10] by the use of the multiple LED estimation model (MLEM). MLEM uses overlap between LED beams to improve the accuracy of proximity-based LED indoor localization systems. Unlike previous proximity-based LED IPS, MLEM provides a platform for improving the accuracy of indoor positioning irrespective of the size of the enclosed area considered. MLEM takes advantage of the simplicity of proximity based positioning algorithms to develop prototypes from off-the-shelf components. The programming of MLEM transmitter system with existing consumer electronics based infrared protocols (IRPs) allows the creation of a low powered communication link between LED transmitters and an off-theshelf infrared (IR) receiver.

Depending on the application for which it is used, popular infrared protocols are either set up in simplex or serial half duplex configurations [11], [12]. The half-duplex configuration by the infrared data association (IrDA) defines standards for point-to-point communication between infrared devices. These devices are designed to allow remote host-to-host communication. Consumer electronics based infrared (CEI) protocols are designed for point-to-point simplex communication links. Unlike the IrDA protocols, CEI protocols do not have specified standards and, thus, the protocol design varies from one manufacturer to another. Nevertheless, despite their nonstandardization, CEI protocols tend to be particularly inexpensive and require simple hardware for their implementation. In addition, existing software libraries make implementation of CEI based protocols easier. The major limitation of CEI protocols is their low transmission rates. However, MLEM requires only a few bits for localization. Therefore, CEI protocols are a suitable choice in MLEM positioning. In addition, since LED-based positioning only requires positional 
data from the transmitter and not vice versa, investigations in this work are narrowed down to the CEI protocols.

CEI protocols use a frame based synchronization mechanism for transmission and detection of data between the transmitter and the receiver. Literature survey and searches returned thirteen popular CEI protocols which include the Sony, NEC, Samsung, JVC, Panasonic, Phillips (RC5 and RC6), Phillips RC-MM multimedia, Mitsubishi, LG, Aiwa, Denon, and Sharp protocols $[13]-[16]$. A study of these protocols reveals a generic pattern of packet formation and this is illustrated in Fig. 1 where some fields may not be present depending upon the specific protocol used. The pre-pulse and starts bits pattern are used to inform the receiver of the point at which it is to start reading in various forms of pulse rise or fall delays that represent ones or zeros depending on the particular encoding scheme used.

\begin{tabular}{|c|c|c|c|c|c|c|}
\hline $\begin{array}{c}\text { Pre- } \\
\text { pulse or } \\
\text { lead in }\end{array}$ & $\begin{array}{c}\text { Start } \\
\text { bit(s) }\end{array}$ & Address bits & $\begin{array}{c}\text { Command or } \\
\text { data bits }\end{array}$ & $\begin{array}{c}\text { Post } \\
\text { pulse or } \\
\text { lead out }\end{array}$ & Stop bits & $\begin{array}{c}\text { Repeat } \\
\text { Identifier }\end{array}$ \\
\hline
\end{tabular}

Fig. 1. Generic structure of an IRP.

The encoding schemes used in all the CEI protocols could be one of four categories which include pulse position modulation (PPM), pulse width modulation (PWM), biphase or Manchester coding (BPC) and pulse distance encoding (PDE). These schemes are designed to ensure error free point-to-point simplex data transmission in electronic products.

Despite the simplicity and suitability of CEI protocols, in the MLEM overlap regions, the behaviour of CEI protocols is uncertain since the protocols are developed for point-topoint optical communication. In the overlapping region of the MLEM system, however, CEI protocols are subject to a multipoint-to-point communication link.

In this paper, we examine the applicability of the various existing IR protocols (IRPs) and investigate their performance in the overlapping region of the MLEM transmitter/receiver system. We then propose a new protocol to deal with overlap while keeping it simple and easy to implement since existing protocols do not meet the required positioning time. Pulse duration multiplexing (PDM), proposed in [10] and [17], is used to investigate the effects of encoding schemes in the overlapping region as it relates to probability of collision and positioning time.

The rest of the paper is organized as follows: a description of the system used to carry out the investigations is presented in Section II. In Section III various encoding schemes in CEI protocols are discussed. A new protocol is developed in Section III-E. Results of the investigations are given in Section IV Finally, conclusions are presented in Section V.

\section{SySTEM DESCRIPTION}

In this section, the indoor LED orientation and the transmitter system with which the different CEI protocols are investigated is examined. The scenario considered contains an overlapping region where signals from two LED light sources meet as illustrated in Fig. 2 Detecting overlap improves positioning accuracy by creating more regions for localization [10]. In Fig. 2, three regions are decoded by the receiver instead of two.

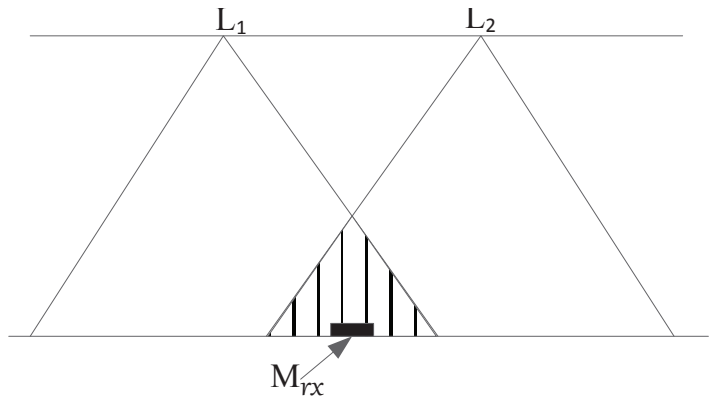

Fig. 2. Illustration of overlapping region for CEI protocol investigation.

In Fig. 2. $\mathrm{L}_{1}$ and $\mathrm{L}_{2}$ are two LEDs in an enclosed space transmitting their positional coordinates and $\mathrm{M}_{r x}$ is a mobile receiver that is located in the overlapping region. For symmetry, $\mathrm{L}_{1}$ and $\mathrm{L}_{2}$ are identical LEDs and their properties are given in Table I] $\mathbf{M}_{r x}$ is a photodetector based receiver in the region under investigation and its properties are also given in Table [1

The transmitter is an infrared based LED system that encapsulates an LED identity (LED-ID) in a frame, encodes the frame using one of the four encoding schemes and modulates the encoded data. The data is subsequently transmitted by the LED. The LED-ID carries the coordinates of the LEDs which is used to infer the receiver position.

TABLE I

PARAMETERS FOR IR TRANSMitTER AND RECEIVER

\begin{tabular}{ll}
\hline \multicolumn{2}{c}{ Parameters } \\
\hline Light emitting diode (LED) & \\
semi-angle of half power $\Phi_{1 / 2}$ & $\pm 35^{\circ}$ \\
peak wavelength $\lambda_{p}$ & $860 \mathrm{~nm}$ \\
total radiant flux $\phi_{a}$ & $70 \mathrm{~mW}$ \\
rise and fall time $t_{r}, t_{f}$ & $12 \mathrm{~ns}$ \\
Photodetector (PD) & \\
Directivity $(\theta)$ & $45^{\circ}$ \\
Peak wavelength $\lambda_{p}$ & $950 \mathrm{~nm}$ \\
Minimum irradiance $E_{(e m i n)}$ & $0.12 \mathrm{~mW} / \mathrm{m}^{2}$ \\
Detector physical area & $1 \mathrm{~cm}{ }^{2}$ \\
Refractive index $n$ & 1.5 \\
Field of Vision $\varphi_{c}$ & $60^{\circ}$ \\
Microcontroller & $\mathrm{ATmega} 328$ \\
Clock frequency & $20 \mathrm{MHz}$ \\
\hline
\end{tabular}

\section{ENCODING SCHEMES PERFORMANCE IN THE OVERLAPPING REGION}

LED-IDs, represented in bits, are encapsulated in packets according to Fig. 1. All the mentioned encoding schemes used in CEI protocols can be represented by rectangular pulse waves in which either the duty cycle or the position of the pulses for ones and zeros differentiates one scheme from another. This encoded data is then modulated to a higher carrier frequency 
using on-off-keying (OOK) due to its simplicity. The carrier frequency for CEI protocols is between $30 \mathrm{kHz}$ and $50 \mathrm{kHz}$. The modulated signal is sent through a driver to the LEDs to generate optical signals. If $v(t)$ is the instantaneous value of a waveform of the pulse with a period $\tau$ and signal levels $v_{\text {min }}$ and $v_{\max }$, then the average value of the waveform of $v(t)$ for a single pulse is given by

$$
v_{\text {avg }}=\frac{1}{\tau} \int_{0}^{\tau} v(t) d t
$$

given the duration of $v_{\min }$ and $v_{\max }$ as $t_{o f f}$ and $t_{o n}$ respectively where $t_{o n}+t_{o f f}=\tau$,

$$
\begin{gathered}
v_{a v g}=\frac{1}{\tau}\left[\int_{0}^{t_{o n}} v_{\max } d t+\int_{0}^{t_{o f f}} v_{\min } d t\right] \\
v_{a v g}=\frac{t_{o n}}{\tau} v_{\max }+\frac{t_{o f f}}{\tau} v_{\min }
\end{gathered}
$$

The power consumption for transmission is proportional to this quantity $v_{a v g}$. Each encoding scheme applied to the overlapping region is therefore represented in subsequent sections as a function of the parameters in (3).

\section{A. Pulse Distance Encoding}

In this encoding scheme, the delay (distance) between the pulses is used to map symbols as shown in Fig. 3a and Fig. 3b. The CEI protocols that makes use of PDE include the JVC, NEC and Sharp protocols [18]. This encoding offers the advantage of regulated power consumption as the duration with which the LED is on is constant irrespective of the bits considered. The delay between pulses used to decode bits makes this encoding scheme susceptible to error. Therefore, protocols which use this technique generally increase the amount of bits to be transmitted by injecting a level of redundancy to correct for errors. This phenomenon can be observed in the NEC and RCA protocols where address, inverse of address, data and inverse of data are transmitted in every frame.

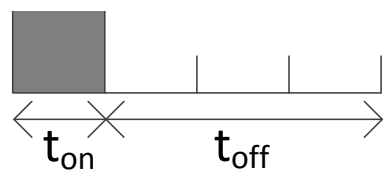

(a) PDE for one

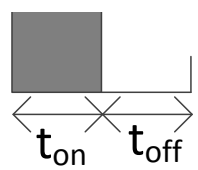

(b) PDE for zero
Fig. 3. General PDE representation of bits for CEI protocols

\section{B. Pulse Width Modulation based encoding}

Pulse width encoding varies the width of the pulse based on the data bit as shown in Fig. 4a and Fig. 4b. Unlike PDE, the variation in the pulse width of PWM makes the power consumption in PWM protocols higher. In order to compensate for this effect, CEI protocols that use this modulation techniques reduce the bits in transmission. Decoding reads the time between pulse rising and falling which makes PWM less susceptible to distortions by noise than PDE protocols. A popular CEI protocol which uses this encoding technique is the Sony SIRC [14].

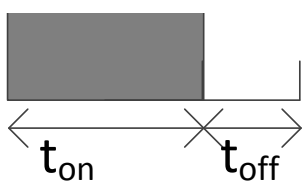

(a) PWM for one

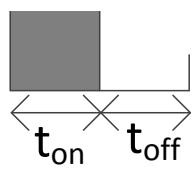

(b) PWM for zero
Fig. 4. General PWM representation of bits for CEI protocols

\section{Biphase encoding}

In this protocol, either a one is represented by a pulse then a delay and a zero by a delay then a pulse or vice versa as illustrated in Fig. 5a and Fig. 5b. This encoding protocol conserves power and also does not use long delays which could corrupt data. In addition the equal periods for ones and zeros could be used as an error detecting measure in decoding. Phillips RC5, RC6 and Nokia NRC are common CEI protocols that use this encoding technique [16].

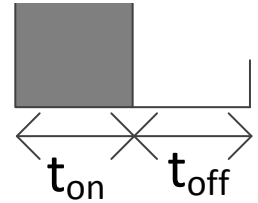

(a) BPC for one

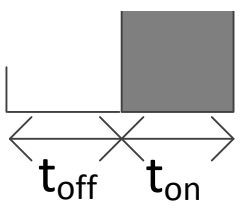

(b) BPC for zero
Fig. 5. General BPC representation of bits for CEI protocols

\section{Pulse Position Modulation}

Of the studied CEI protocols, only the Phillips RC-MM multimedia IR protocol uses pulse position modulation. Unlike other encoding protocols, the PPM CEI protocol uses a 4PPM representation and therefore data is not just represented in $1 \mathrm{~s}$ and $0 \mathrm{~s}$ but in $00 \mathrm{~s}, 01 \mathrm{~s}, 10 \mathrm{~s}$, and $11 \mathrm{~s}$. In this encoding scheme, the position of the pulse determines the information it represents.

In order to evaluate the performance of each CEI protocol based encoding scheme in the overlapping region, their performances in the overlapping region are examined based on the probability of packet collision and positioning time. LED identity data from the two LEDs are encoded in each of these schemes. The encoded data is then arranged in packets based on the protocol(s) that makes use of the encoding scheme. These packets are subsequently modulated using OOK and transmitted through the LEDs. This results in a nonnegative signal from each of the LEDs. Let $x_{1}(t)>0$, represent the instantaneous optical power from $\mathrm{L}_{1}$, the average transmitted power of the optical signal $P_{t 1}$ is given in [11] by:

$$
P_{t 1}=\lim _{\tau \rightarrow \infty} \frac{1}{2 \tau} \int_{-\tau}^{\tau} x_{1}(t) d t
$$

In the same way, $P_{t 2}$ is obtained from LED $\mathrm{L}_{2}$. Assuming a channel model $H$, in this region, the received power from the $\mathrm{PD}$ receiver can be written as

$$
P_{r x}=H_{1} P_{t 1}+H_{2} P_{t 2}
$$


In MLEM, the packets are transmitted with the same carrier frequency, with the same wavelength, at the same time and in the same space (overlapping region). Transmitted packets are therefore subject to collision. Under this condition, with the similarity in channel model from the two transmitters, the probability of packet collision is 1 . Pulse duration multiplexing (PDM), proposed in [10] is used to reduce the probability of collision. Theoretically, in a 2-LED overlapping region and for a given duty cycle $D$, the probability of collision $P_{c}$ is given in [10] by $P_{c}=2 D$.

1) Simulation algorithm for determination of Probability of collision:

a. Given an n-bit LED-ID for LED $\mathrm{L}_{1}$, the data is encoded using one of the four encoding schemes as explained in Section III

b. The encoded data is encapsulated in a packet based on the protocol structure given in Fig 1

c. The data carrying packet is modulated using OOK to a carrier frequency $f$.

d. Using PDM, the packets are multiplexed on a low transmission duty cycle $D$, where $D=$ packettime $/$ (packettime + delay $)[10]$.

e. The process is repeated with LED data for $\mathrm{L}_{2}$.

f. The optical signals are transmitted from both LEDs $N_{t x}$ times.

g. The signals are demodulated and decoded.

h. The number of non-collided packets, $N_{n c}$, and number of collided packets, $N_{c}$ are counted.

i. The probability of collision is given by $\frac{N_{c}}{N_{n c}+N_{c}}$ which is the same as $\frac{N_{c}}{N_{t x}}$.

$\mathrm{j}$. The process is repeated varying $1 / D$ (inverse of duty cycle) from 2 to 50.

$\mathrm{k}$. The process is repeated for other encoding schemes.

The evaluated comparison of probabilities of collision for the different encoding schemes are presented in Section IV-A.

Positioning time is the time the receiver takes to identify its location. In non-overlapping regions, the receiver identifies position by interpreting the data in the packet received. However, in the overlapping region, the receiver is exposed to data from different sources and therefore after decoding the data from a particular source, the receiver does not state the decoded position as its location but rather, the receiver waits to gather positional information from all available transmitters in the overlapping region and then decodes this information. After arranging the encoded data in packet frames, if the time to transmit the frame is $t_{f}$, PDM embeds the frame in low duty cycle pulses by transmitting the frame in time $t_{f}$ and then having a longer delay or off time $t_{o f}$ given by

$$
t_{o f}=\frac{(1-D) t_{f}}{D}
$$

where $D$ is the duty cycle. The time to transmit a frame varies depending on the protocol used for encoding and the number of ones and zeros in the LED identity data. For simulations, for a given encoding scheme, we use the protocol that has the shortest length of frame size and the frame time is taken as the average between the time to transmit a frame with a LED identity data of all ones and that with a LED identity data of all zeros (considering even probability of ones and zeros).

In a region of $N$ LED transmitters, the positioning time, if there is no possibility of collision, is given by $N t_{f}$ s. However in the overlapping region, PDM guarantees a sequence of noncollided packets after a packet is lost in collision by spreading data in the low duty cycle, $D$ [17]. Therefore, the positioning time in this region $t_{p}$ is given by

$$
t_{p}=\left(\frac{N}{D}+1\right) t_{f}
$$

Based on the system model shown in Fig. 2 $N=2$ and therefore $t_{p}=\left(\frac{2}{D}+1\right) t_{f}$.

After decoding all the positional information, the receiver predicts its location as the central point which is common to all the transmitters from which it received positional information. The performances of different encoding schemes based CEI protocols in terms of positioning time $t_{p}$ and inverse of duty cycle when simulated using MATLAB ${ }^{\circledR}$ are illustrated in Fig. 6 Measurements are taken in terms of the inverse of duty cycle because it is proportional to the positioning time as shown in Fig. 6

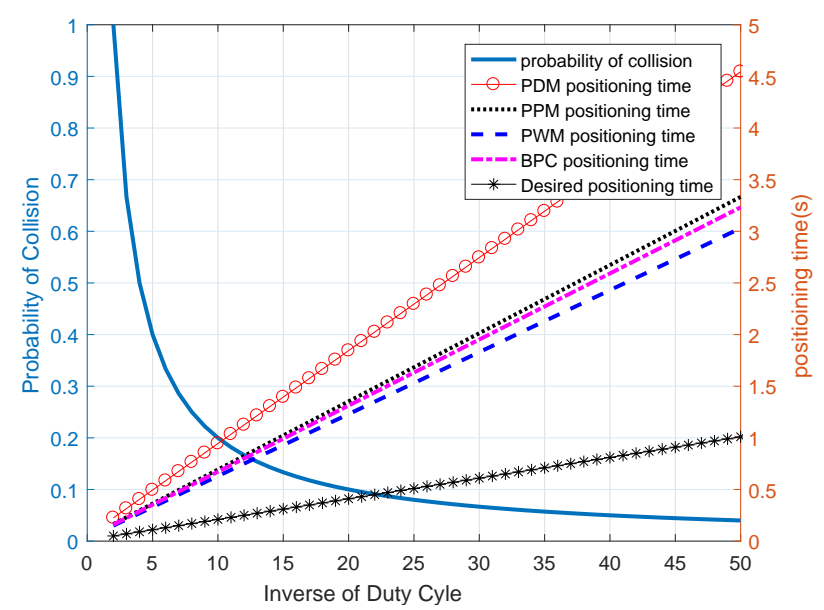

Fig. 6. Performance of various encoding schemes with regards to positioning time and probability of collision.

\section{E. Design of new IRP}

The performances of the CEI protocols, as presented in the results from Fig. 6, do not suffice for real time tracking. Equation (7) reveals that the positioning time is proportional to the frame size of packets from the protocols. Therefore, in order to achieve the desired system, a protocol that allows shorter frame size is designed and its experimental performance is reported in Section IV-B The protocol design rationale is given subsequently and the frame structure for this IRP is shown in Fig. 7 .

\section{1) Rationale behind the MLEM protocol frame design:}

a. Unlike in CEI devices, no button press down event occurs with LED light in MLEM, therefore there is no need for the repeat section of the IR packet. 
b. The protocol is to be embedded in a PDM scheme hence the lead outs and stop bit(s) become redundant and are therefore removed.

c. LEDs transmit their positional information, consequently, the data or command to be transmitted is the LED positional information thereby removing the need for a address, command combination.

d. 4 bits of information can represent up to 15 different light sources and in a standard room (with arbitrary dimension of $5 \mathrm{~m}$ by $5 \mathrm{~m}$ ), the conventional practice is not to use up to 15 different light sources. Therefore the number of bits in the packets is reduced to 6 (two extra bits for wider spaces or future increase in luminaries in an enclosure).

e. The start bits section of the packets are not removed as this signifies the start of frame synchronization.

f. MLEM receivers are designed to operate in power saving modes. Therefore the pre-pulse and start combination is used to wake up the receiver. If the receiver is in its sleep mode and an accidental pulse, which does not match the pre-pulse and start combination, is detected by the receiver, such pulses are ignored.

\begin{tabular}{|c|c|c|}
\hline $\begin{array}{c}\text { Pre- } \\
\text { pulse }\end{array}$ & Start bits & Positional data bits \\
\hline
\end{tabular}

Fig. 7. Designed infrared protocol for MLEM overlapping region

\section{Results AND Discussions}

In this section, results of the investigations of the various encoding scheme based CEI protocols in the overlapping region are presented in two forms. First are the results obtained via simulation using the MATLAB ${ }^{\circledR}$ communications toolbox. To quantify this results, the probability of packet collision is presented and compared in theory and simulation. Then secondly, simulation and experimental comparisons of positioning times for different protocols are given.

\section{A. Simulation Results and discussions}

By carrying out pulse duration multiplexing on the pulses, the probability of collision for the four encoding schemes are presented in Fig. 8a, Fig. 8b, Fig. 8c and Fig. 8d

Irrespective of the encoding techniques used, the figures reveal that pulse duration multiplexing works for all situations in the overlapping region. However, compared to the other figures, Fig. 8d shows that 2-bit pulse position modulation (2PPM) has the lowest deviations from the PDM based theoretical expectation. This is due to the presence of multiple bit symbol encoding in 2PPM compared to other techniques. Although, the different encoding schemes have different amounts of deviation, they all follow the theoretical prediction of the PDM scheme and therefore are all applicable in the MLEM overlapping region.

The positioning time of encoding schemes from different CEI protocols are shown in Fig. 6. In the figure, the NEC protocol and other PDE based protocols have the highest

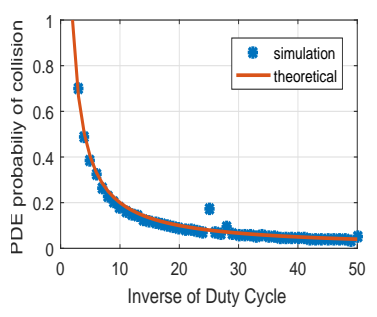

(a) pulse distance encoding

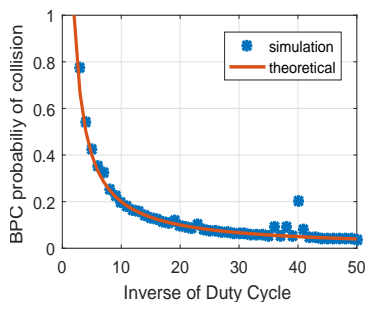

(c) biphase coding

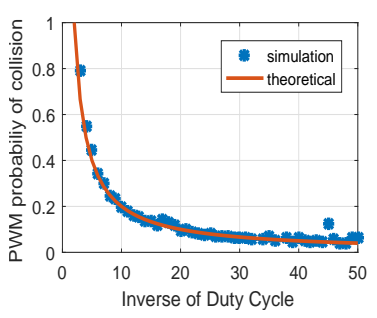

(b) Pulse width modulation

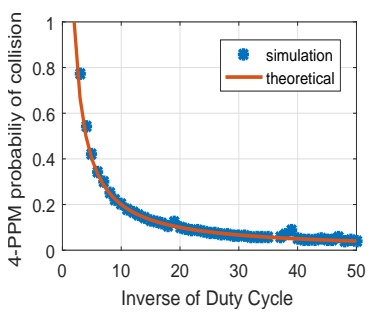

(d) pulse position modulation
Fig. 8. Probability of collision for different encoding schemes in overlap region

positioning times and at low duty cycles, it can be as high as $4.5 \mathrm{~s}$. Considering the low power consumption of PDE based protocols, this protocol is suitable for systems where the object whose position is to be found (target object) is stationary. Examples of applicability of this includes the monitoring of equipment in an hospital, factory, laboratory or an educational institution. PPM, PWM and BPC have moderately better positioning time where positioning is done once every $3 \mathrm{~s}$. These protocols show improvement in positioning time but do not suffice for positioning of mobile targets. Since the average walking rate is approximately $1 \mathrm{~m} / \mathrm{s}$ [19], for mobile targets, indoor positioning is desired every second and therefore an average positioning time of $1 \mathrm{~s}$ is derived for a desired protocol that is suitable for the overlapping region of the MLEM.

\section{B. Experimental Results and discussions}

In this section, the outcomes of the experimental development of a CEI based protocol that suits the MLEM system is given.

Based on the performance of encoding schemes in the overlapping region (Section [III), PWM is preferred as the encoding scheme for this protocol since it performs well for the overlapping region and is less susceptible to error in data transmission.

The designed protocol was implemented in hardware using ATmega 328 microcontrollers with specifications given in Table [1 The positioning time from the experiment is shown in Fig. 9 From this figure, the experimental system performed poorly when packets are multiplexed at high duty cycles (low inverse of duty cycle) and performs better at low duty cycles. This is because at high duty cycles there is a higher probability of collision and most packets are lost in collision. Consequently, more time is required for positioning updates whereas as the duty cycles becomes larger, the probability of collision is lower and the system performs better. At low duty cycles, the result of experimental investigation shows that for 


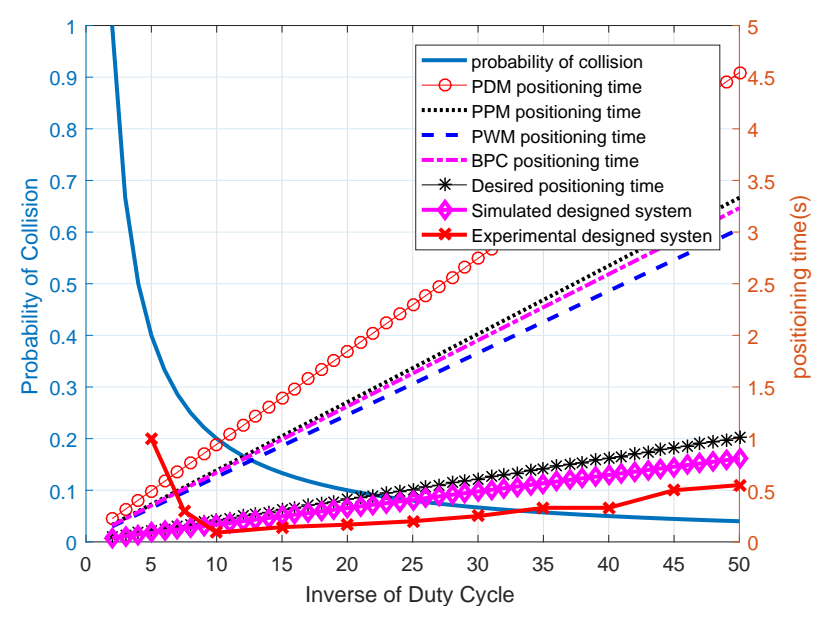

Fig. 9. Performance comparison of the desired positioning time and the designed positioning time.

any region of two overlapping LED beams, a positioning time of $0.5 \mathrm{~s}$ is achieved. This value surpasses the target for indoor positioning of a moving person.

\section{CONCLUSION}

In this paper, the applicability of various encoding schemes from consumer electronics infrared protocols to the multiple LED estimation model (MLEM) for indoor positioning is investigated. The encoding schemes performed as expected in the overlapping region of MLEM but caused significant delays which increased the positioning time. A new protocol is therefore developed that suits the conditions for MLEM design. This protocol is designed to accommodate for energy saving, work on low complexity microcontrollers and accommodate multiple light sources in an indoor location. The protocol is shown to perform better in the overlapping region and the positioning time is reduced from $4.5 \mathrm{~s}$ to $0.5 \mathrm{~s}$. In conclusion, when applied to MLEM, it supports the development of a low cost, energy saving, and low positioning time IPS.

As future work, optimal pulse width for the protocol is designed by minimizing the pulse on and off duration while maintaining bit error rate and optical power constraint.

\section{ACKNOWLEDGMENT}

The authors would like to appreciate the support for this research work from the School of Engineering and Built Environment of Glasgow Caledonian University through the University sponsored research studentship.

\section{REFERENCES}

[1] M. A. Esmail and H. A. Fathallah, "Indoor visible light communication without line of sight: investigation and performance analysis," Photonic Network Communications, vol. 30, no. 2, pp. 159-166, 2015.
[2] S.-H. Yang, H.-S. Kim, Y.-H. Son, and S.-K. Han, "Three-dimensional visible light indoor localization using AOA and RSS with multiple optical receivers," Journal of Lightwave Technology, vol. 32, no. 14, pp. 2480-2485, 15 July 2014.

[3] J. Vongkulbhisal, B. Chantaramolee, Y. Zhao, and W. S. Mohammed, "A fingerprinting-based indoor localization system using intensity modulation of light emitting diodes," Microwave and Optical Technology Letters, vol. 54, no. 5, pp. 1218-1227, 2012.

[4] K. Dividis, Design and Prototyping of a Visible Light Indoor Positioning System. Citeseer, 2007.

[5] M. A. Elkarim, N. A. Mohammed, and M. H. Aly, "Exploring the performance of indoor localization systems based on VLC-RSSI, including the effect of NLOS components using two light-emitting diode lighting systems," Optical Engineering, vol. 54, no. 10, pp. 105 110-105110, 2015.

[6] C. Wang, L. Wang, X. Chi, S. Liu, W. Shi, and J. Deng, "The research of indoor positioning based on visible light communication," China Communications, vol. 12, no. 8, pp. 85-92, 2015.

[7] H.-S. Kim, D.-R. Kim, S.-H. Yang, Y.-H. Son, and S.-K. Han, “An indoor visible light communication positioning system using a RF carrier allocation technique," Journal of Lightwave Technology, vol. 31, no. 1, pp. 134-144, 1 January 2013.

[8] T. Huang, X. Gao, Y. Guo, S. Li, Q. Li, C. Li, H. Zhu, and Y. Wang, "Visible light indoor positioning fashioned with a single tilted optical receiver," in 14th International Conference on Optical Communications and Networks (ICOCN), Nanjing, 3-5 July 2015, pp. 1-4.

[9] K. Kaemarungsi and P. Krishnamurthy, "Modeling of indoor positioning systems based on location fingerprinting," in Twenty-third Annual Joint Conference of the IEEE Computer and Communications Societies (INFOCOM), vol. 2. IEEE, 7-11 March 2004, pp. 1012-1022.

[10] O. Popoola, F. Ogunkoya, W. Popoola, R. Ramirez-Iniguez, and S. Sinanović, "Indoor localization based on multiple LEDs position estimation," in Signal Processing Advances in Wireless Communications (SPAWC), 2016 IEEE 17th International Workshop on. IEEE, 2016, pp. $1-6$.

[11] J. M. Kahn and J. R. Barry, "Wireless infrared communications," Proceedings of the IEEE, vol. 85, no. 2, pp. 265-298, 1997.

[12] I. Millar, M. Beale, B. J. Donoghue, K. W. Lindstrom, and S. Williams, "The IrDA standards for high-speed infrared communications," The Hewlett-Packard Journal, vol. 49, no. 1, pp. 10-26, 1998.

[13] Vishay Semiconductors, "Datasheet: TSFF5510 High Speed Infrared Emitting Diode, $870 \mathrm{~nm}$, GaAlAs Double Hetero," Retrieved Dec. 27, 2008 from http://www.vishay.com/docs/81835/tsff5510.pdf, Sep. 2008.

[14] S. L. Augmented, "Application note: Implementation of transmitters and receivers for infrared remote control protocols with mcus of the stm $32 \mathrm{fO}$ and stm32f3 series," Retrieved October 12, 2016 from www.st.com, March 2016.

[15] A. O. Other, "Ir remote control codes," Retrieved October 12, 2016 from http://read.pudn.com/downloads157/sourcecode/embed/701593/docs/IR, October 2001.

[16] K. Digital, "KD HDMS series IR protocol," Retrieved October 12, 2016 from http://www.keydigital.com, October 2010.

[17] O. R. Popoola, W. O. Popoola, R. Ramirez-Iniguez, and S. Sinanovic, "Optimization of duty cycles for LED based indoor positioning system," in 2016 International Conference for Students on Applied Engineering (ICSAE), Oct 2016, pp. 368-372.

[18] Vishay Semiconductors, "Infrared Emitters," Retrieved Jan. 25, 2011 from http://www.vishay.com/ir-emitting-diodes/.

[19] H. Albrecht, C. Wötzel, L. Erasmus, M. Kleinpeter, N. König, and W. Pöllmann, "Day-to-day variability of maximum walking distance in ms patients can mislead to relevant changes in the expanded disability status scale (EDSS): average walking speed is a more constant parameter," Multiple Sclerosis, vol. 7, no. 2, pp. 105-109, 2001. 